\title{
Knotting and unknotting of phase singularities: Helmholtz waves, paraxial waves and waves in $2+1$ spacetime
}

\author{
M V Berry and M R Dennis \\ H H Wills Physics Laboratory, University of Bristol, Tyndall Avenue, Bristol BS8 1TL, UK
}

Received 13 August 2001

Published 12 October 2001

Online at stacks.iop.org/JPhysA/34/8877

\begin{abstract}
As a parameter $a$ is varied, the topology of nodal lines of complex scalar waves in space (i.e. their dislocations, phase singularities or vortices) can change according to a structurally stable reconnection process involving local hyperbolas whose branches switch. We exhibit families of exact solutions of the Helmholtz equation, representing knots and links that are destroyed by encounter with dislocation lines threading them when $a$ is increased. In the analogous paraxial waves, the paraxial prohibition against dislocations with strength greater than unity introduces additional creation events. We carry out the analysis with polynomial waves, obtained by long-wavelength expansions of the wave equations. The paraxial events can alternatively be interpreted as knotting and linking of worldlines of dislocation points moving in the plane.
\end{abstract}

PACS numbers: 03.65.Vf, 02.10.Kn, 02.40.-k, 41.20.Jb, 42.25.-p, 42.55.-f

\section{Introduction}

In space, the zero lines of complex scalar waves $\Psi(r)=\Psi(x, y, z)$ are their phase singularities, also called wave dislocations (Nye and Berry 1974, Nye 1999) or vortices. They have been studied in optics (Vasnetsov and Staliunas 1999, Soskin 1998, Soskin and Vasnetsov 2001), and in quantum mechanics (Riess 1970a, b, 1987, Hirschfelder et al 1974, Bialynicki-Birula et al 2000). It has long been known that dislocation lines can be closed (Nye and Berry 1974), and recently it was shown that they can be knotted and linked: we (Berry and Dennis 2001a, hereinafter called I) constructed exact solutions of the Helmholtz equation representing torus knots and links; a straightforward application of the ideas in I (Berry 2001) led to knotted and linked dislocation lines in stationary states of electrons in hydrogen.

As a parameter (hereinafter called $a$ ) is varied, the topology of dislocation lines can change, leading to the creation of knots and links from initially simple dislocation loops, and the reverse processes of unknotting and unlinking. Our main purpose here is to elucidate the mechanism of these changes of topology (section 2), and illustrate them with explicit calculations (sections 4 
and 5). In this paper, all our waves are solutions of monochromatic wave equations, that is, stationary waves, and $a$ is an external parameter that could be manipulated experimentally. However, $a$ could represent time, and then the analogous solutions of time-dependent wave equations would describe knotting and linking events in the history of the wave.

We also have two subsidiary aims. The first is to draw attention to families of exact 'polynomial wave' solutions (section 3) of the Helmholtz and paraxial wave equation. These were introduced by Nye (1998), who constructed them by inspection. Here we present a systematic and easy way to generate polynomial waves of several different types, using a longwavelength expansion (this is the opposite of expansions based on geometrical optics, and so could be termed 'anti-geometrical' or 'anti-semiclassical'). The second aim is to clarify a potential confusion about knots and links in paraxial waves, that might have been caused by our previous paper. In I, we constructed knots and links in the Helmholtz equation by perturbing a high-order dislocation loop, and showed that the method fails paraxially because the paraxial wave equation prohibits loops with strength greater than unity. However, as Courtial and Padgett have pointed out to us (private communication), the paraxial prohibition does not prevent the formation of paraxial knots and links by other means, and in section 5 we show precisely how this occurs.

Polynomials do not represent physically realistic beams. Our reason for employing them is to give the analytically simplest local description of dislocation reconnection events in knots and links. However, since the events are structurally stable, they also occur in waves that could be created experimentally, for example the Laguerre-Gauss beams of paraxial optics. In the appendix we give explicit formulae for superpositions of Laguerre-Gauss beams for which knots and links exist over parameter ranges that we specify numerically.

The techniques we use here for creating knots and links are different from those of Poston and Winfree (unpublished), who were studying waves in excitable media. Our methods are based on exact stationary solutions of wave equations, and lead to knots and links threaded by multistranded helices. Theirs are based on algebraic geometry, and lead to functions that are not stationary solutions of wave equations but need not be threaded.

In constructing our explicit examples, it is convenient to introduce a predominant phase factor, specifying a preferred direction, and also to fix the wavenumber $k=1$, equivalent to measuring distances in units of wavelength/ $(2 \pi)$. Thus we write

$$
\begin{aligned}
& \Psi(\boldsymbol{r}) \equiv \psi(\boldsymbol{R}, z) \exp (\mathrm{i} z) \\
& \boldsymbol{R} \equiv(x, y)(\text { Cartesian coordinates }) \equiv(R, \phi) \text { (cylindrical coordinates })
\end{aligned}
$$

Dislocation lines are defined by $\psi(\boldsymbol{R}, z)=0$.

For waves $\Psi_{\mathrm{H}}$ satisfying the Helmholtz equation, $\psi=\psi_{\mathrm{H}}$ satisfies

$$
\nabla_{\perp}^{2} \psi_{\mathrm{H}}+2 \mathrm{i} \partial_{z} \psi_{\mathrm{H}}+\partial_{z}^{2} \psi_{\mathrm{H}}=0 \quad \nabla_{\perp}^{2} \equiv \partial_{x}^{2}+\partial_{y}^{2} .
$$

In the paraxial approximation, the second derivative term in $z$ is dropped, and waves $\Psi_{\mathrm{p}}$, when rephased according to (1) to give $\psi_{\mathrm{p}}$, satisfy the paraxial or parabolic wave equation

$$
2 \mathrm{i} \partial_{z} \psi_{\mathrm{p}}=-\nabla_{\perp}^{2} \psi_{\mathrm{p}}
$$

This form of writing emphasizes the well known fact that the paraxial approximation to the three-dimensional wave equation is exactly the time-dependent Schrödinger equation in two spatial dimensions $\boldsymbol{R}$, with $z$ representing time. Therefore the paraxial knots and links we will generate also represent topologically interesting histories of nodes in evolving quantum waves for free particles in the plane. 

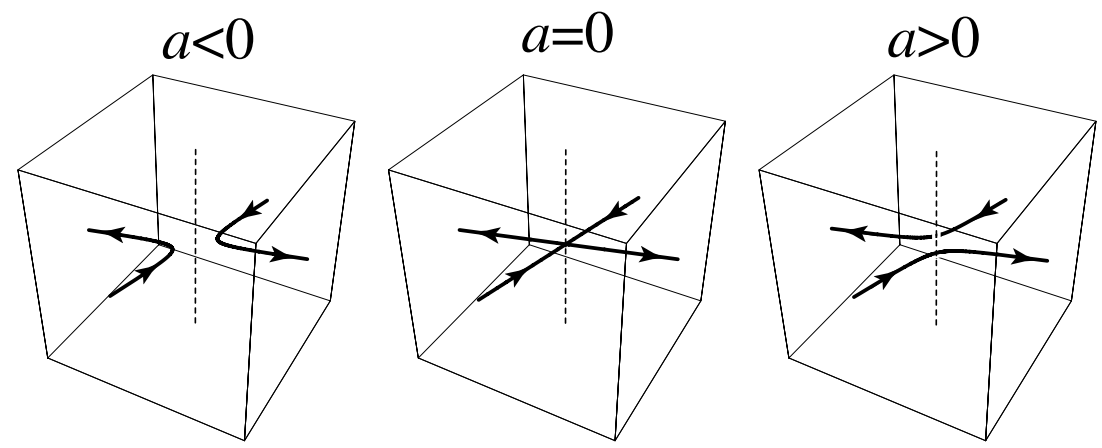

Figure 1. Dislocation reconnection, described by (4) with $\gamma=\pi / 12$. Arrows give the directions about which the phase increases right-handedly, and the dashed line indicates $N=0$ (equation (5)).

\section{Local dislocation reconnection}

The topology of dislocation loops can change if increasing $a$ causes two strands to cross and reconnect as illustrated in figure 1 . A wave with this property is

$$
\psi=\frac{x y}{\sin 2 \gamma}-\frac{1}{2}\left(x^{2}+y^{2}\right)+a+\mathrm{i} z .
$$

This is a solution of both the Helmholtz and paraxial equations (2) and (3), describing two dislocation lines in the form of hyperbolas lying in the $x, y$ plane, with asymptotes in directions $\phi=\gamma, \pi / 2-\gamma, \pi+\gamma, 3 \pi / 2-\gamma$, whose branches switch as $a$ passes through zero. Geometrically, this is the behaviour of contour lines of a function $(\operatorname{Re} \psi)$ near a saddle.

At a reconnection, the direction $N$ of a dislocation line (Berry and Dennis 2000) becomes indeterminate, in addition to $\psi$ vanishing, so that

$$
\psi(\boldsymbol{r}, z)=0 \quad \boldsymbol{N}(\boldsymbol{R}, z)=\operatorname{Im} \nabla \psi^{*} \times \nabla \psi=2 \nabla \operatorname{Re} \psi \times \nabla \operatorname{Im} \psi=0 .
$$

$N$ is the vector product of two real vectors, and so vanishes when these vectors are parallel. This corresponds to two conditions, so generically $\boldsymbol{N}=0$ on lines in space. (These lines are also 'L lines' (Nye and Hajnal 1987) where $\nabla \psi$, regarded as a complex vector field, is linearly polarized (Berry and Dennis 2001b).) Reconnection occurs when a dislocation line encounters an $N=0$ line, typically as a single parameter varies. In the wave (4), $N=0$ on the $z$ axis. Later we will find that the conditions (5) are very convenient in locating the reconnection events.

A different and very simple way that might be envisaged for one or more dislocation loops to change topology is for two strands to cross inertly, that is, without the reconnection just described. It is not difficult to construct functions whose line zeros do cross inertly. For example,

$$
\psi=(x+\mathrm{i} z)(y+\mathrm{i}(z-a))
$$

represents a dislocation along the $y$ axis and a dislocation parallel to the $x$ axis that crosses the first when $a=0$. However, this simple product is unstable against perturbation: even the addition of a (possibly complex) constant causes the dislocation crossing to take place via two reconnection events like those in figure 1, rather than inertly. Moreover, (6) is not a solution of the wave equation (2) or (3), and our attempts to construct solutions by adding higher terms were frustrated because the additional terms destroyed the crossing. Bialynicki-Birula et al (2000) exhibit a wave resembling (6) and satisfying the time-dependent Schrödinger equation 
Table 1. Polynomials $R^{-m} \exp (\mp \mathrm{i} m \phi) \psi_{\mathrm{H} m n}$ associated with Bessel beam solutions of the Helmholtz equation.

\begin{tabular}{ll}
\hline$n=0$ & 1 \\
$n=1$ & $R^{2}+2 \mathrm{i}(m+1) z$ \\
$n=2$ & $R^{4}-4(m+2)(m+1) z^{2}+4 \mathrm{i}(m+2) z\left(R^{2}-(m+1)\right)$ \\
$n=3$ & $R^{6}-12(m+3)(m+2) z^{2}\left(R^{2}-2(m+1)\right)$ \\
& $+2 \mathrm{i}(m+3) z\left(3 R^{4}-2(m+2)\left(3 R^{2}+2(m+1)\left(z^{2}-3\right)\right)\right)$ \\
\hline
\end{tabular}

in three dimensions, representing the interaction of two dislocations, with time acting as the parameter. However, close examination again reveals two reconnection events of the type described by the normal form (4), confirming that the sequence shown in figure 1 is stable against perturbation and therefore occurs naturally, as we will see later in several examples.

For completeness, we should mention that there are other types of interactions between dislocations satisfying wave equations (Nye and Berry 1974), though these are not relevant in the present context: loops that shrink and disappear, and straight parallel dislocation lines that approach and annihilate or bounce off each other.

\section{Polynomial waves}

To generate our knots and links in I, we used superpositions of Bessel beams, and the same construction would also produce the topology changes that we are exploring here. However, we can get algebraically simpler descriptions of the same phenomena by expanding $\psi_{\mathrm{H}}$ and $\psi_{\mathrm{p}}$ in series of polynomials. This is consistent with the fact that dislocations, regarded as singularities, describe the fine structure of wavefields, and are complementary to the caustic singularities of geometrical optics (Berry 1981, 1998). Although the intensity of polynomial waves increases in directions transverse to the $z$ axis, so that they cannot represent beams globally, they are admirably suited to describe not only the local transformations of dislocations (indeed, this is why they were introduced by Nye (1998)), but also the 'semi-local' topology we study here.

To generate these polynomials, we start with the Bessel beams with angular momentum $\pm m$ and transverse wavenumber $\kappa$. Exact Bessel beam solutions of the Helmholtz equation (2) are

$\psi_{\mathrm{H}, m}(R, \phi, z)=\exp ( \pm \mathrm{i} m \phi) J_{m}(\kappa R) \exp \left\{\mathrm{i} z\left(\sqrt{1-\kappa^{2}}-1\right)\right\} \quad m \geqslant 0$.

For the paraxial wave equation (3), the corresponding beams are

$$
\psi_{\mathrm{p}, m}(R, \phi, z)=\exp ( \pm \mathrm{i} m \phi) J_{m}(\kappa R) \exp \left\{-\frac{1}{2} \mathrm{i} z \kappa^{2}\right\} \quad m \geqslant 0 .
$$

The algorithm producing polynomial waves is based on the observation that if the solutions (7) and (8) are expanded in powers of $\kappa$ (this is the anti-geometrical expansion) then the coefficients of $\kappa^{n}$ are also solutions of the corresponding equations. These coefficients, denoted $\psi_{m n}$, are polynomials in $R$ and $z$, multiplied by exp $( \pm \mathrm{i} m \phi)$. Tables 1 and 2 list the first few polynomials for Helmholtz and paraxial waves, normalized so that the coefficient of the highest power of $R$ is unity. For $n \geqslant 2$, the paraxial polynomials have fewer terms than their Helmholtz counterparts, and we will see that this makes a crucial difference to the dislocation topology. (For $m=0$, the Helmholtz polynomials reduce to linear combinations of those listed by Nye (1998).)

It is worth remarking that polynomial waves can be generated by the long-wavelength expansion of any set of solutions of (2) and (3), not just the Bessel beams that are useful for 
Table 2. Polynomials $R^{-m} \exp (\mp \mathrm{i} m \phi) \psi_{\mathrm{p} m n}$ associated with Bessel beam solutions of the paraxial wave equation.

\begin{tabular}{ll}
\hline$n=0$ & 1 \\
$n=1$ & $R^{2}+2 \mathrm{i}(m+1) z$ \\
$n=2$ & $R^{4}-4(m+2)(m+1) z^{2}+4 \mathrm{i}(m+2) z R^{2}$ \\
$n=3$ & $R^{6}-12(m+3)(m+2) z^{2} R^{2}$ \\
& $+2 \mathrm{i}(m+3) z\left(3 R^{4}-4(m+1)(m+2) z^{2}\right)$ \\
\hline
\end{tabular}

our present purposes. For example, expansion of the simple Helmholtz and paraxial plane waves

$\psi_{\mathrm{H}}=\exp \left\{\mathrm{i}\left(\kappa x+\left(\sqrt{1-\kappa^{2}}-1\right) z\right)\right\} \quad$ and $\quad \psi_{\mathrm{p}}=\exp \left\{\mathrm{i}\left(\kappa x-\frac{1}{2} \kappa^{2} z\right)\right\}$

provides a way of systematically generating the Cartesian polynomial waves of Nye (1998).

\section{Knotting and linking of Helmholtz waves}

To create knots and links that will then be made to change topology, we first adopt the strategy already employed and explained in detail in I. In brief, we create a wave $\psi_{0}$ with an axial dislocation of strength $p$ encircled by a dislocation ring of strength $q$, and then perturb this with a wave of amplitude $a$ that does not possess dislocations threading the ring. When $a$ is small, the result is that the ring explodes, acquiring the topology of a torus knot with winding numbers $(p, q)$ if $p$ and $q$ are coprime, and a link otherwise; the axial threading dislocation explodes into $p$ twisted curves resembling a $p$-stranded helix.

To get the topology change, we simply increase the 'perturbation' $a$. Then, as we will see, the torus on which the knot or link is wound, and the cylinder on which the $p$ threading strands are wound, get thicker until they encounter each other. At this moment, the threading strands swallow the knot, by the mechanism of section 2, and the knot disappears.

We first illustrate this procedure in the simplest case: the link $p=q=2$ (in mathematics this is called the Hopf link). For the central strength 2 dislocation, we use polynomial waves from table 1 with $m=2$. A strength 2 ring in the symmetry plane $z=0$ requires

$$
\psi_{0}\left(R_{\mathrm{c}}, \phi, 0\right)=\partial_{R} \psi_{0}\left(R_{\mathrm{c}}, \phi, 0\right)=\partial_{z} \psi_{0}\left(R_{\mathrm{c}}, \phi, 0\right)=0
$$

for some radius $R_{\mathrm{c}}$. These equations can be satisfied by adjusting the constants $A$ and $B$ in the linear combination

$$
\psi_{0 \mathrm{H}, \text { link }}=N\left(\psi_{\mathrm{H} 20}+A \psi_{\mathrm{H} 21}+B \psi_{\mathrm{H} 22}\right)
$$

where $N$ is a constant, to be chosen so that the coefficient of the highest power of $R$ is unity. An easy calculation gives

$$
A=-\frac{1}{6} \quad B=\frac{1}{144} \quad R_{\mathrm{c}}=\sqrt{12} .
$$

For the perturbation, the simplest choice is the plane wave $\psi=a$, leading to the total wave

$$
\psi_{\mathrm{H}, \mathrm{link}}=\exp (2 \mathrm{i} \phi) R^{2}\left[\left(R^{2}-12\right)^{2}-48 z^{2}+16 \mathrm{i} z\left(R^{2}-12\right)\right]+a .
$$

Figure 2(a) shows the link that has been created from the ring as $a$ increases from zero; this is already familiar from our earlier analysis in I. To see what happens for larger $a$, we calculate the vector field $N(\boldsymbol{R}, \boldsymbol{z})$, whose vanishing at a dislocation signals a change of topology (cf (5)). 

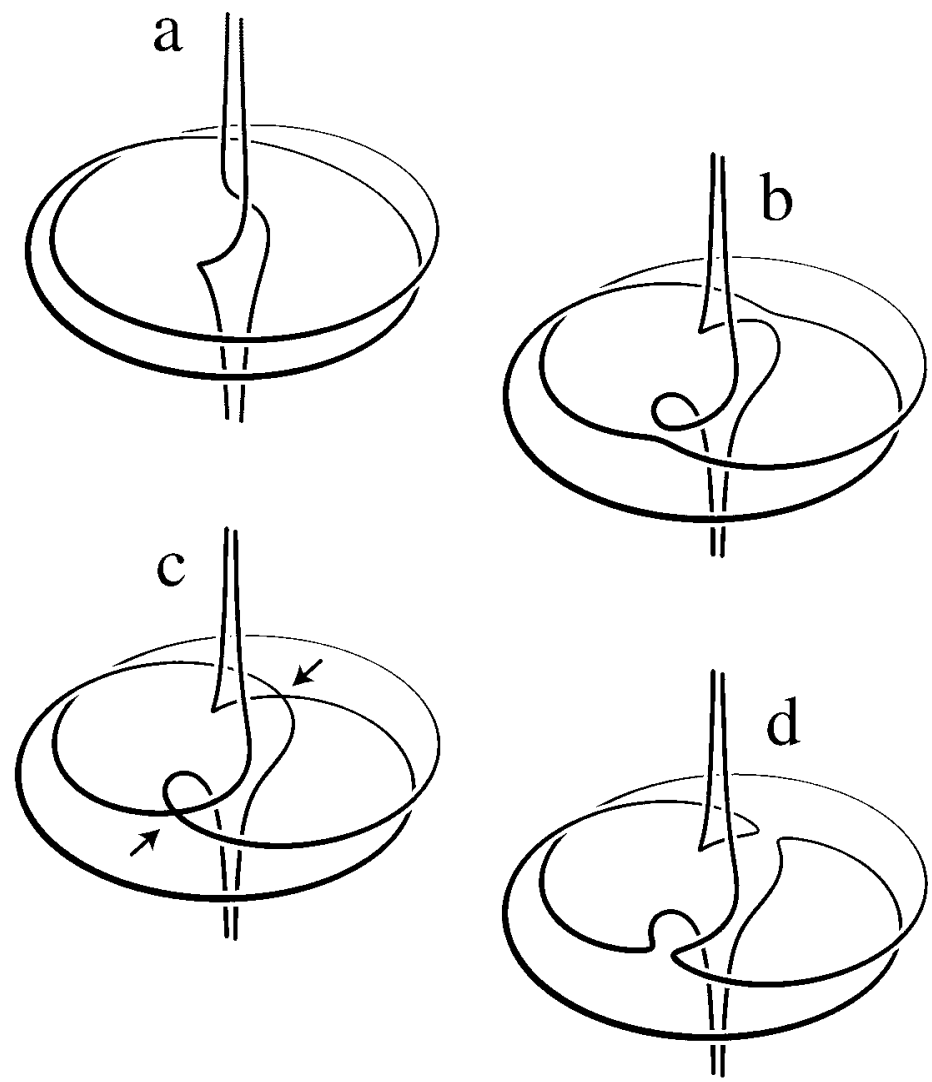

Figure 2. Creation and destruction of linked dislocations in a Helmholtz wave as $a$ increases, computed from (13). (a) $a=100$, just after the creation of the link; (b) $a=250 ;(c)$ $a=a_{\text {crit }}=256$, when reconnection destroys the link; $(d) a=260$. (For clarity, these figures have been magnified in the $z$ direction.) To draw the figures, a multibranched representation of the zero lines of (13), parametrized by $\phi$, was used, to depict points on the dislocation line as a series of overlapping dots, computed using Mathematica, whose sizes decreased away from the observer. Then the knots were connected into smooth curves, and the crossings of dislocation strands clarified, using Adobe Illustrator.

Since $N$ depends only on derivatives of $\psi$, this vector is independent of $a$ for the wave (11). Direct calculation from (5) gives

$$
\begin{aligned}
& N_{R}=-64 R^{4} z\left[5\left(R^{2}-12\right)^{2}+144 z^{2}\right] \\
& N_{\phi}=-192 R^{3}\left(R^{2}-4\right)\left[\left(R^{2}-12\right)^{2}+48 z^{2}\right] \\
& N_{z}=16 R^{3}\left[\frac{3}{4}\left(R^{2}-12\right)^{3}\left(R^{2}-4\right)+80 z^{2}\left(72-18 R^{2}+R^{4}\right)+576 z^{4}\right] .
\end{aligned}
$$

Inspection shows that $N$ vanishes along the $z$ axis $(R=0)$, and on the rings $(R, z)=$ $(\sqrt{ } 12,0)$ and $(2,0)$. On the $z$ axis and on the ring $(\sqrt{ } 12,0), \psi=a$, so that these lines correspond to the birth at $a=0$ of the link and its two threading dislocations. On the ring $(2,0),(13)$ gives

$$
\psi_{\mathrm{H}, \mathrm{link}}(2, \phi, 0)=256 \exp (2 \mathrm{i} \phi)+a .
$$

This represents the topology change we are interested in, which therefore occurs at

$$
a_{\text {crit }}=256 \quad R_{\text {crit }}=2 \quad \phi_{\text {crit }}=\frac{1}{2} \pi \text { and } \frac{3}{2} \pi \quad z_{\text {crit }}=0 .
$$


Figures $2(b)-(d)$ show the sequence through $a_{\text {crit }}$. The link is destroyed by two simultaneous reconnection events of the type shown in figure 1 . (The fact that the reconnections occur simultaneously is an artefact of the symmetry of the wave (13), and does not compromise the genericity of the separate reconnections; for a slightly more general perturbation, e.g. $a+\varepsilon x+\delta y$ for complex $\varepsilon$ and $\delta$, the reconnections would happen at different values of $a$.) Note how as $a$ approaches $a_{\text {crit }}$ the threading dislocations distort, in preparation for the reconnection that requires the connecting dislocations to be locally coplanar, according to the model in section 2 .

Our second example is the trefoil knot $p=3, q=2$. The analysis is very similar. Instead of (11), we have a linear combination of the Helmholtz polynomial waves $\psi_{\mathrm{H} 30}, \psi_{\mathrm{H} 31}$, and $\psi_{\mathrm{H} 32}$, with coefficients determined again by (10), leading to the analogue of (13):

$$
\psi_{\mathrm{H}, \mathrm{knot}}=\exp (3 \mathrm{i} \phi) R^{3}\left[\left(R^{2}-20\right)^{2}-80 z^{2}+20 \mathrm{i} z\left(R^{2}-20\right)\right]+a .
$$

When $a=0$, the knot is created from the ring at $(\sqrt{ } 20,0)$. Calculation of $N$ gives the analogue of the formulae (14), showing that the topology change occurs when the knot encounters the $\operatorname{ring}(\sqrt{ }(60 / 7), 0)$. The analogue of $(15)$ is

$$
\psi_{\mathrm{H}, \mathrm{knot}}\left(\sqrt{\frac{60}{7}}, \phi, 0\right)=\frac{768000}{342} \sqrt{\frac{15}{7}} \exp (3 \mathrm{i} \phi)+a .
$$

Topology change occurs at the analogue of (16), namely

$$
\begin{array}{lcc}
a_{\text {crit }} & =\frac{768000}{343} \sqrt{\frac{15}{7}}=3277.66 \ldots & R_{\text {crit }}=\sqrt{\frac{60}{7}} \\
\phi_{\text {crit }}=\left( \pm \frac{1}{3} \pi, \pi\right) & z_{\text {crit }}=0 . &
\end{array}
$$

Now the knot is destroyed by three reconnections (again simultaneous for inessential reasons of symmetry).

\section{Knotting and linking of paraxial and Schrödinger waves}

As shown in I, it is impossible to create higher-order dislocation loops in paraxial waves. Therefore an attempt to make knots and links by analogy with the previous section, using the paraxial polynomial waves from table 2 instead of the Helmholtz polynomials of table 1, ought to fail. In fact it does not fail, and detailed study not only illustrates the topology changes we are focusing on here but also provides an instructive example of how the 'paraxial prohibition' works in practice.

Let us consider the paraxial analogue of the link construction based on (11), namely

$$
\psi_{0 \mathrm{p}, \text { link }}=N\left(\psi_{\mathrm{p} 20}+A \psi_{\mathrm{p} 21}+B \psi_{\mathrm{p} 22}\right)
$$

and attempt to satisfy the three conditions (10). Using table 2 we find that the first two conditions, $\psi=0$ and $\partial_{R} \psi=0$, imply $A=-2 / R^{2}, B=A^{2} / 4$, but that it is impossible to satisfy $\partial_{z} \psi=0$. Inspecting the resulting unperturbed wave, namely

$$
\psi_{0 \mathrm{p}, \text { link }}=\exp (2 \mathrm{i} \phi) R^{2}\left[\left(R^{2}+\frac{2}{A}\right)^{2}-48 z^{2}+16 \mathrm{i} z\left(R^{2}+\frac{3}{2 A}\right)\right],
$$

we see that the absence, in the paraxial polynomial labelled $\psi_{\mathrm{p} 22}$, of one term that is present in the corresponding Helmholtz polynomial, implies that changing $A$ trivially rescales the whole pattern: the framework of paraxial waves is too rigid for all the equations (10) to be satisfied simultaneously. Therefore, the value of $A$ is immaterial, and, simply to give the same critical ring radius as for the Helmholtz wave (13), we choose $A=-1 / 6$, giving

$$
\psi_{\mathrm{p}, \text { link }}=\exp (2 \mathrm{i} \phi) R^{2}\left[\left(R^{2}-12\right)^{2}-48 z^{2}+16 \mathrm{i} z\left(R^{2}-9\right)\right]+a \text {. }
$$



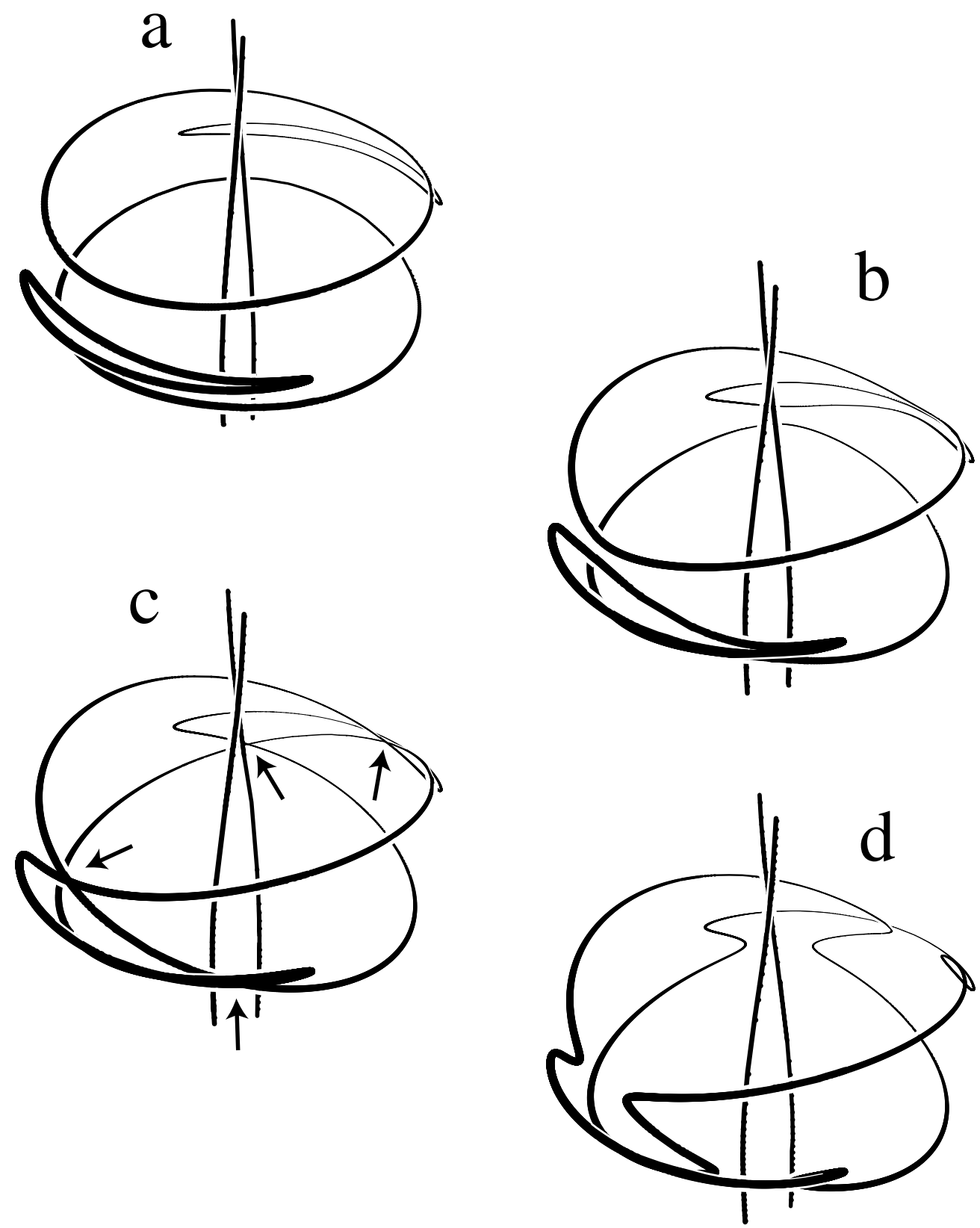

Figure 3. Creation and destruction of linked dislocations in the paraxial wave as $a$ increases, computed from (22). (a) $a=8.6$; (b) $a=34.56$; (c) $a=a_{\text {crit1 }}=37.82 \ldots$, when reconnection creates the link; $(d) a=41.5 ;(e) a=172.8 ;(f) a=250.6 ;(g) a=a_{\text {crit } 2}=256$, when a second reconnection destroys the link; $(h) a=259.2$. (For clarity, these figures have been magnified in the $z$ direction.) The figures were drawn as described in the caption to figure 2 , but with $R$ as parameter.

The only difference between (22) and (11) is the final numerical coefficient -9 instead of 12 - but this is a crucial difference. Instead of the single strength 2 dislocation ring of 

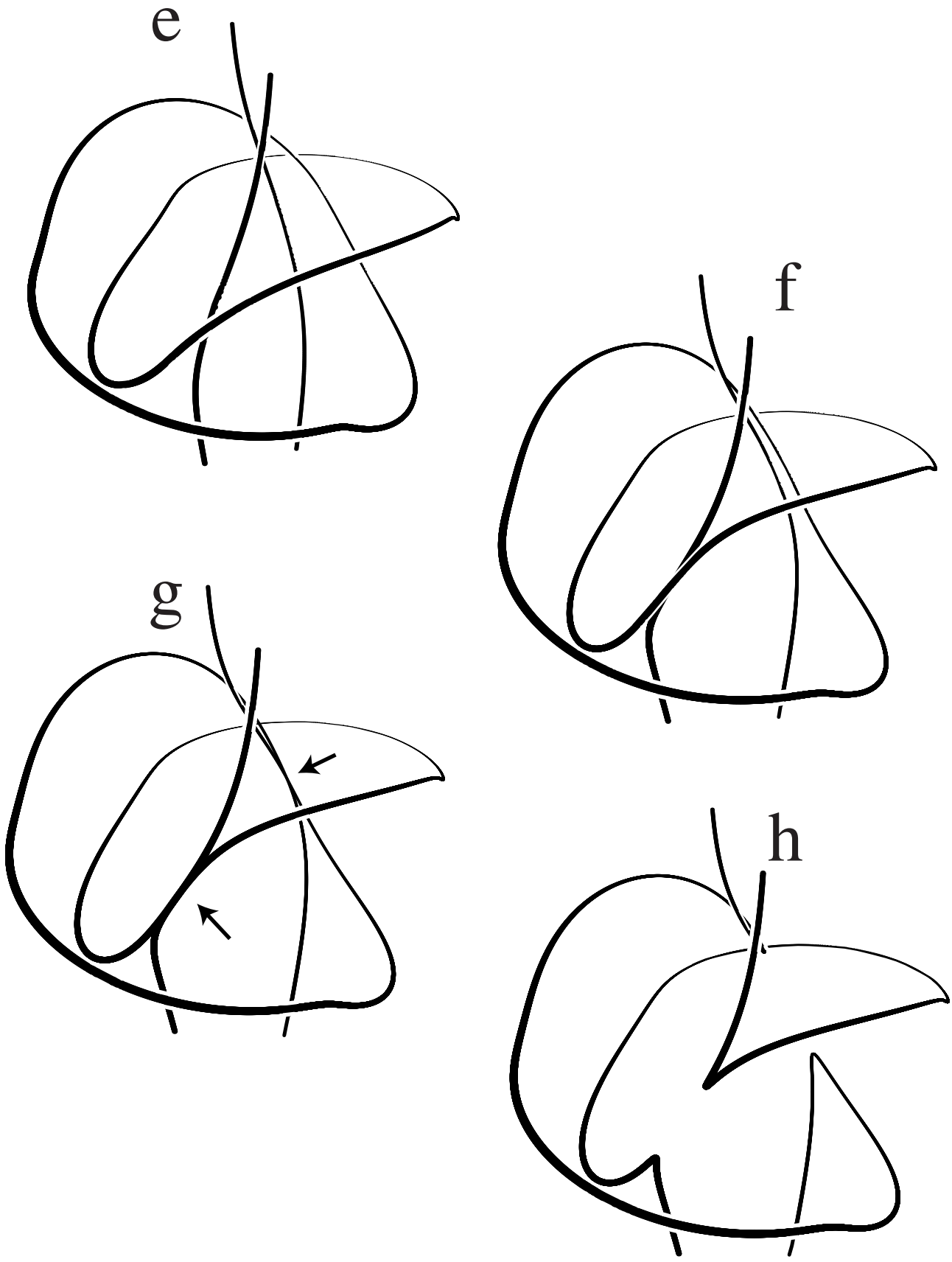

Figure 3. (Continued.)

the Helmholtz wave, the paraxial wave has three rings: a strength zero ring, at $(\sqrt{ } 12,0)$, and two rings with strength 1 , at $(3, \pm 1 / 4)$. Again, there is a central threading dislocation of strength 2 . Under perturbation ( $a$ increasing from zero), the ring with strength zero becomes two thin crescent-shaped loops lying close to the $x y$ plane (figure $3(a)$ ). 
To understand what happens when $a$ is further increased, we need the vector $N$ corresponding to the wave (22). From (5),

$$
\begin{aligned}
& N_{R}=-64 R^{4} z\left[5 R^{4}-72 R^{2}+216+144 z^{2}\right] \\
& N_{\phi}=-192 R^{3}\left[\left(R^{2}-4\right)\left(R^{2}-9\right)\left(R^{2}-12\right)+48 z^{2}\left(R^{2}-3\right)\right] \\
& N_{z}=4 R^{3}\left[3\left(R^{2}-12\right)^{3}\left(R^{2}-4\right)+240 z^{2}\left(72-18 R^{2}+R^{4}\right)+2304 z^{4}\right] .
\end{aligned}
$$

As before, $N$ vanishes along the $z$ axis $(R=0)$, associated with the birth of the threading dislocations near the $z$ axis, and on the ring $(\sqrt{ } 12,0)$, corresponding to the birth of the crescents. $N$ also vanishes on the ring $(2,0)$, which as we will see plays a similar role as in the Helmholtz case.

However, there are two additional $N=0$ rings, with no counterpart in the Helmholtz case, at

$$
\begin{aligned}
& R_{\text {crit } 1}=\sqrt{2+4 \sqrt{2} \cos \mu}=3.15637 \ldots \\
& z_{\text {crit1 }}= \pm \frac{1}{6} \sqrt{-43+52 \sqrt{7} \cos \mu-70 \cos 2 \mu}= \pm 0.187059 \ldots
\end{aligned}
$$

where

$$
\mu=\frac{1}{3}\left(\pi-\arccos \frac{47}{28 \sqrt{7}}\right)
$$

These correspond to the creation of a link, by four simultaneous interactions of the two dislocation crescents with the two strength 1 dislocation rings that were present at $a=0$. The process is shown in figures $3(b)-(d)$. The parameter value $a_{\text {crit1 }}$ and the azimuths $\pm \phi_{\text {critl }}$ of these reconnection events are obtained by inserting the values (24) into (22):

$$
a_{\text {crit } 1}=37.8161 \ldots \quad \phi_{\text {crit } 1}=1.13987 \ldots=65.31^{\circ} .
$$

Two of these interactions are with the upper ring, at $+z_{\text {crit } 1}$ and $\phi_{\text {crit } 1}$ and $\pi+\phi_{\text {crit } 1}$, and two are with the lower ring, at $-z_{\text {crit1 }}$ and $-\phi_{\text {crit1 }}$ and $\pi-\phi_{\text {crit } 1}$.

What we learn from this is that although the paraxial prohibition prevents the formation of higher-strength dislocation rings that would allow the link to be created by perturbation from $a=0$, for a sufficiently large 'perturbation' the link forms anyway (figure 3(e)), though by a different mechanism, and thereafter the dislocation topology is the same for paraxial as for Helmholtz waves. We can see this by further increasing $a$ : just as in the Helmholtz case, the link is eventually destroyed by reconnection with the threading dislocations. For the paraxial link, this second pair of critical events (figures $3(f)-(h)$ ) is associated with the $N=0$ ring at $R=2$; the corresponding parameters are the same as in (16), because the paraxial and Helmholtz waves (22) and (11) are identical when $z=0$.

For the trefoil knot, the analogous sequence is creation of the knot by interaction of three thin crescents with the two pre-existing dislocation rings, at six simultaneous reconnection events, followed by its destruction at three simultaneous reconnections with the three threading dislocation lines. The paraxial analogue of (17) is

$$
\psi_{\mathrm{p}, \mathrm{knot}}=\exp (3 \mathrm{i} \phi) R^{3}\left[\left(R^{2}-20\right)^{2}-80 z^{2}+20 \mathrm{i} z\left(R^{2}-16\right)\right]+a
$$

where again the value $R=\sqrt{ } 20$ is chosen for comparison with the Helmholtz case and has no intrinsic significance. 
For the six creation events, the critical parameter values are

$$
\begin{aligned}
& a_{\text {crit } 1}=475.995 \\
& R_{\text {crit } 1}=4 \sqrt{\frac{5(1+5 \cos \mu)}{21}}=4.160528 \ldots \\
& \phi_{\text {crit1 }}=0.75071 \ldots=43.49^{\circ} \\
& z_{\text {crit } 1}=\sqrt{\frac{500(2 \cos \mu-\cos 2 \mu)-701}{147}}=0.191897 \ldots
\end{aligned}
$$

where

$$
\mu=\frac{1}{3}\left(\pi-\arccos \frac{2194}{3125}\right)
$$

with connections with the upper ring $z=+z_{\text {crit1 }}$ occuring at azimuths $\phi_{\text {crit1 }}, \phi_{\text {crit } 1} \pm 2 \pi / 3$, and connections with the lower ring $z=-z_{\text {crit } 1}$ occuring at azimuths $-\phi_{\text {crit1 }},-\phi_{\text {crit } 1} \pm 2 \pi / 3$. The three destruction events occur at the same parameter values (19) as for the Helmholtz knot.

In the interpretation of the paraxial wave equation as a Schrödinger equation, these families of dislocation lines, parameterized by $a$, take on a different meaning. For fixed $a$, the solutions describe patterns of dislocation points moving in the $x y$ plane and encountering each other in creation and annihilation events. In spacetime, the world lines of the dislocation points are the knots and links we have described. As $a$ varies, the creation and destruction of knots and links appear as pairs of dislocation points bouncing off each other, rather than pairs that are created or annihilate.

\section{Acknowledgments}

We are indebted to Dr Johannes Courtial and Professor Miles Padgett for their paraxial calculations that inspired the work reported here. MRD is supported by a University of Bristol postgraduate scholarship.

\section{Appendix. Knots and links in Laguerre-Gauss beams}

Laguerre-Gauss beams, that is solutions of the paraxial wave equation (3) for wavenumber $k=1$, are

$\psi_{\mathrm{LG} m n}(R, \phi, z)=\left(\frac{w(-z)}{w(z)}\right)^{n} \frac{\exp \left\{-\frac{R^{2}}{2 w(z)}\right\}}{w(z)^{m+1}} R^{m} \exp (\mathrm{i} m \phi) L_{n}^{m}\left(\frac{R^{2}}{|w(z)|^{2}}\right)$

where $L$ denotes the associated Laguerre polynomials and

$$
w(z)=1+\mathrm{i} z
$$

(These formulae describe beams with waist width unity; beams with other widths can be obtained by rescaling $R$ and $z$.)

The simplest link and knot are obtained by superposing three of these beams with a plane wave of amplitude $a$. We choose the superpositions to make the degenerate ring (where $\psi$ and $\partial_{R} \psi$ vanish for $a=0$ ) occur at $R=1$ (cf the remarks before equation (22)). As with the paraxial polynomial waves of section 5 , knots and links are created as $a$ increases through a critical value $a_{\text {crit1 }}$ and destroyed at $a_{\text {crit2 }}$.

For the link, we find

$$
\psi=\psi_{\mathrm{LG} 20}-\frac{6}{7} \psi_{\mathrm{LG} 21}+\frac{2}{7} \psi_{\mathrm{LG} 22}+a .
$$


The link is created at four reconnection events, whose critical parameter values are

$$
\begin{array}{ll}
a_{\text {crit } 1}=0.003370651 \ldots & R_{\text {crit } 1}=0.883533 \ldots \\
z_{\text {crit } 1}= \pm 0.0263448 \ldots & \phi_{\text {crit } 1}= \pm 69.41^{\circ} \text { and } \mp 110.59^{\circ} .
\end{array}
$$

For these Laguerre-Gauss beams, there are four destruction events (unlike the two for polynomial waves), with parameters

$$
\begin{array}{ll}
a_{\text {crit } 2}=0.017968 \ldots & R_{\text {crit } 2}=0.535649 \ldots \\
z_{\text {crit2 }}= \pm 0.0388719 \ldots & \phi_{\text {crit } 2}= \pm 108.05^{\circ} \text { and } \mp 71.95^{\circ} .
\end{array}
$$

For the knot,

$$
\psi=\psi_{\mathrm{LG} 30}-\frac{8}{13} \psi_{\mathrm{LG} 31}+\frac{2}{13} \psi_{\mathrm{LG} 32}+a .
$$

The knot is created at six reconnection events, whose critical parameter values are

$$
\begin{array}{lll}
a_{\text {crit } 1}=0.000972409 \ldots & R_{\text {crit } 1}=0.913606 \ldots \\
z_{\text {crit } 1}= \pm 0.0146031 \ldots & \phi_{\text {crit } 1}=\mp 74.71^{\circ}, \pm 45.29^{\circ}, \pm 165.29^{\circ} .
\end{array}
$$

There are six destruction events, with parameters

$$
\begin{array}{llrl}
a_{\text {crit2 }}=0.00573333 \ldots & & R_{\text {crit2 }}=0.624702 \ldots \\
z_{\text {crit2 }}= \pm 0.0143240 \ldots & \phi_{\text {crit } 2}=\mp 53.58^{\circ}, \pm 66.42^{\circ}, \pm 186.42^{\circ} .
\end{array}
$$

\section{References}

Berry M V 1981 Singularities in waves and rays Les Houches Lecture Series Session 35 ed R Balian, M Kléman and J-P Poirier (Amsterdam: North-Holland) pp 453-543

-1998 Much ado about nothing: optical dislocation lines (phase singularities, zeros, vortices, ...) Singular Optics (SPIE Proc. 3487) ed M S Soskin (Bellingham, WA: SPIE) pp 1-5

2001 Knotted zeros in the quantum states of hydrogen Found. Phys. 31 659-67

Berry M V and Dennis M R 2000 Phase singularities in isotropic random waves Proc. R. Soc. A 456 2059-79

2001a Knotted and linked phase singularities in monochromatic waves Proc. R. Soc. A 457 2251-63 (reference I of the text)

2001b Polarization singularities in isotropic random waves Proc. R. Soc. A 457 141-55

Bialynicki-Birula I, Bialynicka-Birula Z and Sliwa C 2000 Motion of vortex lines in quantum mechanics Phys. Rev. A 61032110

Hirschfelder J O, Goebel C J and Bruch L W 1974 Quantized vortices around wavefunction nodes. II J. Chem. Phys. 61 5456-9

Nye J F 1998 Unfolding of higher-order wave dislocations J. Opt. Soc. Am. A 15 1132-8

1999 Natural Focusing and Fine Structure of Light: Caustics and Wave Dislocations (Bristol: Institute of Physics Publishing)

Nye J F and Berry M V 1974 Dislocations in wave trains Proc. R. Soc. A 336 165-90

Nye J F and Hajnal J V 1987 The wave structure of monochromatic electromagnetic radiation Proc. R. Soc. A 409 21-36

Riess J 1970a Nodal structure, nodal flux fields, and flux quantization in stationary quantum states Phys. Rev. D 2 $647-53$

-1970b Nodal structure of Schrödinger wave functions and its physical significance Ann. Phys., NY 57 301-21

1987 Quantised vortex motion through rings in quantum mechanics J. Phys. A: Math. Gen. 20 5179-88

Soskin M S (ed) 1998 Singular Optics (SPIE Proc. 3487) (Bellingham, WA: SPIE)

Soskin M S and Vasnetsov M V (ed) 2001 Singular Optics (Optical Vortices): Fundamentals and Applications (SPIE Proc. 4403) (Bellingham, WA: SPIE)

Vasnetsov M and Staliunas K (ed) 1999 Optical Vortices (New York: Nova Science) 